\title{
Tangles and Degenerate Tangles
}

\author{
Andres J. Ruiz-Vargas \\ School of Basic Sciences - École Polytechnique Fédérale de Lausanne, Switzerland \\ andres.ruizvargas@epfl.ch \\ http://sb.epfl.ch/
}

\begin{abstract}
We study some variants of Conway's thrackle conjecture. A tangle is a graph drawn in the plane such that its edges are represented by continuous arcs, and any two edges share precisely one point, which is either a common endpoint or an interior point at which the two edges are tangent to each other. These points of tangencies are assumed to be distinct. If we drop the last assumption, that is, more than two edges may touch one another at the same point, then the drawing is called a degenerate tangle. We settle a problem of Pach, Radoičić, and Tóth [7], by showing that every degenerate tangle has at most as many edges as vertices. We also give a complete characterization of tangles.
\end{abstract}

Keywords: thrackles, tangles, degenerate tangles, tangled thrackles, graph drawing, intersection graphs.

\section{Introduction}

A drawing of a graph (or a topological graph) is a representation of the graph in the plane where the vertices are represented by different points of the plane, and the edges by simple continuous curves connecting their corresponding vertices while avoiding any other vertices. When it is clear by the context, we will make no distinction between the drawing of a graph and the graph itself. Similarly, we use $V(G)$ to refer both to the vertices and their corresponding points and $E(G)$ to refer both to the edges and their corresponding curves.

A drawing of $G$ is called a thrackle if every pair of edges meets exactly once, either at an endpoint or at a proper crossing (a proper crossing of two edges is a crossing where one of the edges passes from one side of the other edge to its other side). Conway conjectured, in the sixties, that a thrackle cannot have more edges than vertices 2] [1] 9. Since then, there have been many results. The first, by Lovász, Pach, and Szegedy [6], established that the number of edges of a thrackle with $n$ vertices is at most $2 n-3$. This was improved, by Cairns and Nikolayevsky, to $1.5 n$ [3, and again improved, by Fulek and Pach, to a bit less than $1.428 n$ [4].

In 2011 going along a similar direction, Pach, Radoičić, and Tóth [7] coined the term tangle, degenerate tangle, and tangled thrackle.

\footnotetext{
* Research partially supported by Swiss National Science Foundation grant 200021125287/1 and by Hungarian Science Foundation EuroGIGA Grant OTKA NN 102029 .
} 
Definition 1. A tangle is a graph drawn in the plane such that its edges are represented by continuous arcs, and any two edges share precisely one point, which is either a common endpoint or an interior point at which the two edges are tangent to each other. These points of tangencies are assumed to be distinct.

Definition 2. A degenerate tangle is a graph drawn in the plane such that its edges are represented by continuous arcs, and any two edges share precisely one point, which is either a common endpoint or an interior point at which the two edges are tangent to each other. These points of tangencies are not assumed to be distinct.

The only difference between a tangle and a degenerate tangle is that for the latter, more than two edges may touch one another at the same point.

Definition 3. A tangled thrackle is a topological graph where any two edges shares precisely one point, either an endpoint, a point of tangency, or a proper crossing. These points of tangencies, and crossings, are assumed to be distinct.

Definition 4. A degenerate tangled thrackle is a topological graph where any two edges share precisely one point, either an endpoint, a point of tangency, or a proper crossing. More than two edges may touch at the same interior point, but no crossing and touching point can coincide.

In [7. Pach et al. proved that every tangle with $n$ vertices has at most $n$ edges. And using a lemma from [8], which bounds the number of edges of a simple topological graph with $n$ vertices and no $k$ pairwise disjoint edges, they proved that the number of edges of a tangled thrackle with $n$ vertices is at most $c n \log ^{12} n$, for a fixed constant $c$. It is conjectured, 7] that the number of edges of a tangled thrackle with $n$ vertices is $O(n)$, however none of the current techniques seem to work for this problem. For the case of degenerate tangled thrackles, no bounds are known.

In [7, it is also mentioned that a degenerate tangle with $n$ vertices has at most $3 n-6$ edges. We improve this bound, showing that the number of edges of a degenerate tangle is at most the number of vertices, and this is tight, just as in the case of tangles.

In this paper, in an attempt to further understand the nature of tangles, degenerate tangles, thrackles, and tangled thrackles, we characterize tangles. We say that a graph is a star graph if it is the same as $K_{1, k}$ for some integer $k$. We do not consider graphs with vertices of degree zero.

Theorem 1. A graph $G$ can be drawn as a tangle if and only if $G$ is:

1. a graph with at most four edges; or

2. a graph that can be turned into a star graph by the removal of an edge and some isolated vertices.

Theorem 2. Every degenerate tangle with $n$ vertices has at most $n$ edges. 


\section{Tools and Definitions}

Our main tool of this paper, is the following lemma together with the definition of semi-dual of a tangle.

Lemma 1. Let $\mathcal{D}$ be the family of graphs consisting of the graphs with exactly 5 edges and where every vertex has degree 3 or less. Then, no tangle can contain a graph from $\mathcal{D}$ as a subgraph.

Before proving this Lemma, let us define the semi-dual graph of certain tangles. The semi-dual together with Kuratowski's theorem [5], will be used extensively throughout the proofs of this paper.
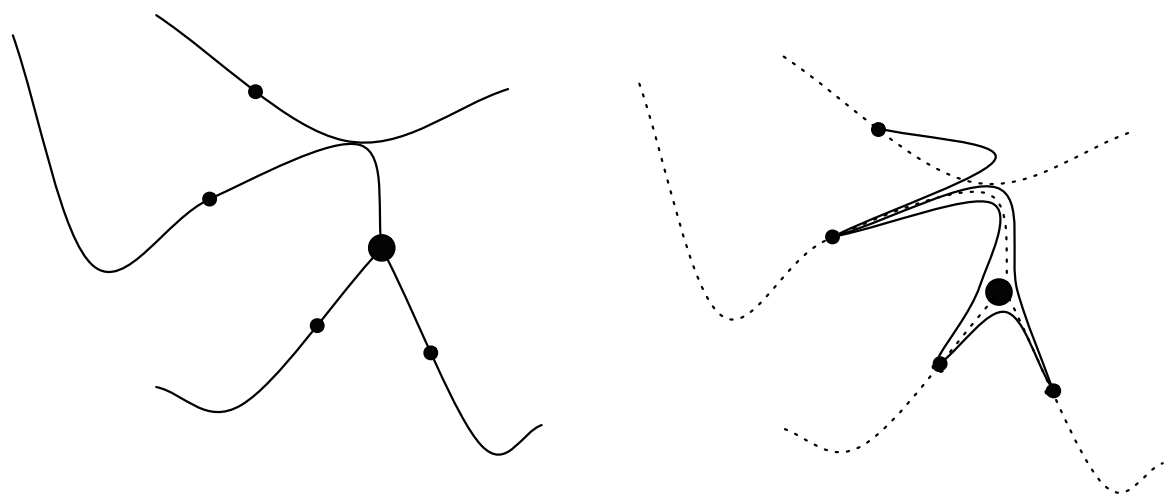

Fig. 1. From left to right: a drawing of a tangle and its semi-dual. The small points represent the fixed points of each edge, while the large point is a vertex.

Consider a tangle $G$ where each vertex has degree 3 or less. For each edge, fix an interior point. For every pair of points, connect them using two pieces of the corresponding curves that meet, either at an endpoint or at a point of tangency. We further perturb this drawing to eliminate overlapping of edges, and hence obtain a planar graph. Note that this can be perturbed into a planar drawing because all vertices have degree three or less, see Figure 1. We call this resulting drawing the semi-dual of $G$ and denote it $G^{s}$.

For a degenerate tangle, we say that $x$ is a degenerate point if it is in the relative interior of at least 3 edges. We say that the edges that meet in $x$, a degenerate point, are its corresponding or adjacent edges. We say that a vertex is adjacent to $x$, if one of its edges passes through $x$; otherwise we say that it is non-adjacent to $x$.

Let us return to Lemma 1 .

Proof. Suppose, for a contradiction, that there exists a tangle $G$ with $H \in \mathcal{D}$ a subgraph of $G$. By deleting all edges of $G$ except the ones in $H$, we are left with a tangle drawing of $H$. Consider the planar graph $H^{s}$, semi-dual of $H$. Then it is clear that $H^{s}$ is a planar drawing of $K_{5}$, which is a contradiction to Kuratowski's theorem. 


\section{Tangles}

In [7] it was proved that every tangle with $n$ vertices has at most $n$ edges. As an application of Lemma 1 we reprove, in a different way to Pach et al., the following theorem.

Theorem 3. [7] Every tangle with $n$ vertices has at most $n$ edges.

Proof. For a contradiction, consider a minimal counterexample, that is, let $G$ be a tangle with $n$ vertices, $n+1$ edges and such that every proper subgraph of $G$ has no more edges than vertices. If $G$ has a vertex of degree 1 , we could delete it, and get a tangle with $n-1$ vertices and $n$ edges, contradicting the minimality of $G$. Hence every vertex of $G$ has degree at least 2 . Therefore, $G$ has one of the following properties:

- 1. All vertices have degree 2, except for a vertex of degree 4.

- 2. All vertices have degree 2, except for 2 vertices of degree 3 .

Case 1

Let $v$ be a vertex of degree 4 . Delete one of its edges. Hence we have a graph where every vertex has degree at most 3 . Note that this graph has at least 5 vertices, hence it has at least 5 edges. This is a contradiction to Lemma 1 .

Case 2

In this case, all vertices have degree at most 3 . Because there are two vertices of degree 3 , then there are at least 4 vertices, hence at least 5 edges, which is again a contradiction to Lemma 1.

\subsection{Proof of Theorem 1}

Proof. Let $G$ be a tangle. If $G$ has a vertex $v$ of degree 3 or more then, by Lemma 1. it follows that there is at most one edge not adjacent to $v$. If all the vertices of $G$ have degree less or equal to 2, then, again by Lemma 1, it follows that it cannot have more than 4 edges.

Graphs with at most four edges and graphs that can be turned into a star graph by the removal of an edge can be drawn as tangles, see Figure 2.
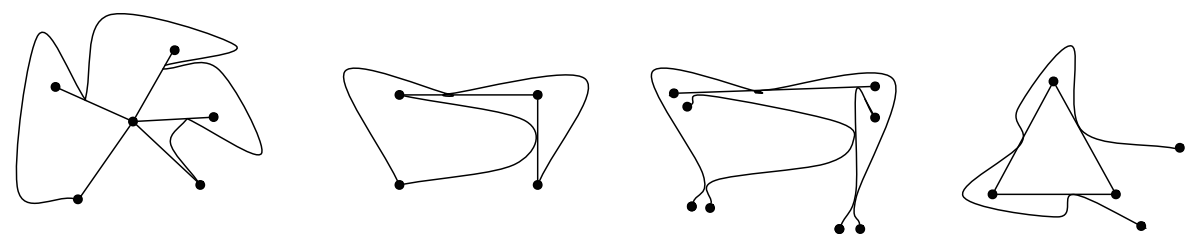

Fig. 2. From left to right, a tangle drawing of a star graph with an extra edge, a cycle of length four, a matching of size four, and a triangle with an extra edge. All four-edge graphs with no cycles can be drawn from the drawing of a matching of size four. 


\section{Degenerate Tangles}

\subsection{Proof of Theorem 2}

Proof. For a contradiction, consider a minimal counterexample, that is, let $G=$ $(V, E)$ be a degenerate tangle with $n$ vertices, $n+1$ edges and such that every proper subgraph of $G$ has no more edges than vertices. Every vertex will have degree at least 2 , since a vertex of degree one could be removed contradicting the assumptions of $G$. Hence, just as in the proof of Theorem 3, there are two cases to consider:

- 1. All vertices have degree 2, except for a vertex of degree 4.

- 2. All vertices have degree 2, except for 2 vertices of degree 3 .

Since $G$ has more edges than vertices, Theorem 3 implies that there exists a degenerate point $x$ where edges $a, b, c \in E$ meet. Denote by $a_{1}, a_{2} ; b_{1}, b_{2} ; c_{1}, c_{2}$ the endpoints of $a, b, c$; respectively. We will show that neither of the two cases is possible. For the latter, we will get a contradiction to Lemma 1 by finding 5 edges that form a tangle, with no vertex having degree greater than three. However, one thing must be noted: because we are now dealing with degenerate tangles, one of the key things is to find such five edges where no three pass through the same point, that is between the 5 edges no degenerate point is made.

\section{Case 1}

Suppose first that $v$, the vertex of degree four, is not adjacent to $x$. Then any three edges from the adjacent edges to $v$, together with any two edges from $\{\mathrm{a}, \mathrm{b}, \mathrm{c}\}$ give a contradiction to Lemma 1. Now, let us consider the case where $v$ is adjacent to $x$, and without loss of generality let us assume that $v=a_{1}$. In this case, edges $b, c$ together with the 3 edges of $v$ that are not adjacent to $x$, give a contradiction to Lemma 1, this is because between those 5 edges no degenerate point is formed.

\section{Case 2}

Let $u$ and $v$ be the vertices of degree 3 . Then, there are at least 5 edges with an endpoint in either $u, v$, or both. These 5 edges are a contradiction to Lemma 1 . again because between those 5 edges no degenerate point is formed.

Acknowledgements. I would like to thank Radoslav Fulek for listening to my ideas while giving me feedback, Gabriel Nivasch for helping write properly and János Pach for asking me the questions that led to these results.

\section{References}

1. Unsolved problems. Chairman: P. Erdős. In: Combinatorics (Proc. Conf. Combinatorial Math., Math. Inst., Oxford, 1972), Inst. Math. Appl., Southend-on-Sea, pp. 351-363 (1972)

2. Brass, P., Moser, W., Pach, J.: Research Problems in Discrete Geometry. Springer, New York (2005) 
3. Cairns, G., McIntyre, M., Nikolayevsky, Y.: The thrackle conjecture for $K_{5}$ and $K_{3,3}$. In: Towards a Theory of Geometric Graphs, Contemp. Math., vol. 342, pp. 35-54. Amer. Math. Soc., Providence (2004)

4. Fulek, R., Pach, J.: A computational approach to Conway's thrackle conjecture. Comput. Geom. 44(6-7), 345-355 (2011)

5. Kuratowski, K.: Sur le probleme des courbes gauches en topologie. Fund. Math. 15, 271-283 (1930)

6. Lovász, L., Pach, J., Szegedy, M.: On Conway's thrackle conjecture. Discrete Comput. Geom. 18, 369-376 (1998)

7. Pach, J., Radoičić, R., Tóth, G.: Tangled thrackles. Geombinatorics (2012) (to appear)

8. Pach, J., Tóth, G.: Disjoint Edges in Topological Graphs. In: Akiyama, J., Baskoro, E.T., Kano, M. (eds.) IJCCGGT 2003. LNCS, vol. 3330, pp. 133-140. Springer, Heidelberg (2005)

9. Ringeisen, R.D.: Two old extremal graph drawing conjectures: progress and perspectives. Congressus Numerantium 115, 91-103 (1996) 